\author{
Military Technical College \\ Kobry Elkobbah, \\ Cairo, Egypt
}

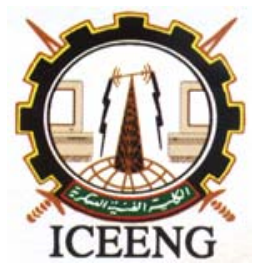

\author{
$5^{\text {th }}$ International Conference \\ on Electrical Engineering \\ ICEENG 2006
}

\title{
COMPUTATIONAL ERRORS OF ANALYTIC COARSE ALIGNMENT FOR STRAP DOWN INERTIAL NAVIGATION SYSTEM (SDINS)
}

\author{
Y. Z. Elhalwagy*, I. I. Arafa ${ }^{* *}$, and K. H. Mostafa ${ }^{* * *}$
}

\begin{abstract}
:
In this paper, two proposed computational methods of coarse alignment for strap down inertial navigation systems (SDINS) are presented. Their associated drift, skew, and scale alignment errors are evaluated analytically. Although the computational formulas for analytic ground alignment are identical in the ideal case, the error characteristics are dependent upon the employed basis. With properly selecting the basis to compute the best estimate of transformation matrix, the drift misalignment angles of analytic alignment can be made to be equivalent to those, which can be found by physical gyro-compassing.
\end{abstract}

Keywords: Inertial sensors, Inertial navigation system, strap down systems, alignment and error analysis

\section{NOMENCLATURE}

\section{Abbreviations}

$\mathrm{SD}=$ strap down

INS = Inertial navigation system

SDINS = strap down inertial navigation system

Symbols

$\bar{g} \quad=$ Local gravity vector.

$\omega_{i e} \quad=$ Earth turning rate.

$\varphi \quad=$ Local geographical latitude.

$\boldsymbol{a}^{\boldsymbol{b}} \quad=$ Measurements of accelerometers in body frame

$\omega^{\boldsymbol{b}} \quad=$ Measurements of gyros in body frame.

$I \quad=$ Identity matrix

$S \quad=$ Alignment error

$\phi \quad=$ Alignment error

\footnotetext{
* Egyptian Armed Forces

${ }^{* *}$ Egyptian Armed Forces

**** Egyptian Armed Forces
} 


\section{INTRODUCTION}

Initial misalignment is one of the major error sources of inertial navigation systems (INS). The initial alignment errors will affect the system error not only in the attitude indication but also in the velocity and position information [1-5]. Therefore, prior to normal navigation, alignment process must be performed to determine the orientation of the platform axes with respect to the navigation coordinate frame. One method for obtaining the initial angular orientation is through the use of an external reference by optical means. However, this method is very limited to operational environment. Alternatively, for most ground-based applications, a self-contained alignment method known as gyro-compassing [2-7] provides another operational approach. As a general rule, gyro-compassing consists of two phases, that is, leveling and azimuth alignment. The basic principle of gyro-compassing consists of feeding signals proportional to the accelerometer outputs or/and velocity error outputs to the appropriate level gyros and azimuth gyro.

The purpose of initial alignment process is to drive the misalignment angles to zero. Unfortunately, this goal can never be achieved in a practical system. This drawback is deduced from the sensor errors, which cannot be compensated ideally. Initial alignment process is of vital important to inertial navigation equipments. Alignment requirement is related to the necessity for the transformation of the sensor outputs into the best estimate of the attitude, (velocity and position), of the vehicle with respect to the reference navigational frame [1-8]. Therefore, poor initial alignment accuracy will end up with poor navigation accuracy. Generally speaking, alignment process is divided into three phases: leveling, coarse, and fine alignment. The leveling mode roughly establishes level attitude by sensing the direction of gravity with respect to the vehicle body axes. Next, the coarse alignment mode determines azimuth to within a few degrees by sensing the rotation of the gravity vector with respect to the inertial space and to provide a fairly good initial condition for the fine alignment processing. Theoretically, an analytical self-alignment method for strap-down systems is functionally equal to the physical gyro compassing in gimbaled systems [9]. The error characteristics of both systems seem to be identical at steady state in a stationary base. An error analysis for an analytic alignment method had been done by Britting [2]. He showed that the drift misalignment angles about north and vertical axes are identical with those obtained with physical gyro compassing, but a difference exists about the east axis. This part stimulates the researchers to study the properties of analytic alignment methods for SDINS. Besides, the application of strap down technology has been attracting considerable attention nowadays.

This paper investigates two analytical/computational methods of coarse alignment for SDINS. The two proposed methods are associated with error analysis. The formulas are evaluated in a computer simulation with a comparison between them. Finally, the paper is terminated by a conclusion section.

\section{Problem Formulation and Formulas of Analytical Alignment}

It is well known that the self-alignment requirement is the measurement of two noncollinear vectors, the local gravity vector $\bar{g}$ and the earth rate $\omega_{i e}$, in both body and navigation frames. The local gravity vector and earth rate can be sensed in the body frame by the inertial sensors, accelerometers and gyros respectively, which are known in the Earthfixed frame $[3,6]$. For the purpose of this investigation, we assume that the navigation axes are aligned with the local-level north, east, and down axes. Then $\boldsymbol{g}$ and $\omega_{i e}$ can be expressed in the navigation frame as: 


$$
\begin{aligned}
& \boldsymbol{g}^{\boldsymbol{n}}=\left[\begin{array}{lll}
0 & 0 & g
\end{array}\right]^{T} \\
& \boldsymbol{\omega}_{\boldsymbol{i e}}^{\boldsymbol{n}}=\left[\begin{array}{lll}
\Omega \cos \varphi & 0 & -\Omega \sin \varphi
\end{array}\right]^{\boldsymbol{T}}
\end{aligned}
$$

Where $\boldsymbol{g}$ and $\Omega$ represent the magnitude of gravity and Earth rate respectively; $\varphi$ is the local geographical latitude.

The analytical alignment problem is to build a set that consists of three linearly independent vectors $\left[\begin{array}{lll}\boldsymbol{V}_{1} & \boldsymbol{V}_{2} & \boldsymbol{V}_{3}\end{array}\right]$ upon body and navigation frames, such that the solution of transformation matrix $C_{b}^{n}$ can be found.

$$
\left[\begin{array}{lll}
\boldsymbol{V}_{1} & \boldsymbol{V}_{2} & \boldsymbol{V}_{3}
\end{array}\right]_{\boldsymbol{n}}=C_{b}^{n}\left[\begin{array}{lll}
\boldsymbol{V}_{1} & \boldsymbol{V}_{2} & \boldsymbol{V}_{2}
\end{array}\right]_{\boldsymbol{b}}
$$

In general, there are six possible sets, which can be used for achieving the analytical alignment purpose $[4,5]$. However only the following two simple and significant sets, $\boldsymbol{B}_{1}$ and $\boldsymbol{B}_{2}$ are analyzed here in some detail where $\boldsymbol{B}_{1}$ and $\boldsymbol{B}_{2}$ are defined as:

$$
\begin{aligned}
& \boldsymbol{B}_{1}=\left[\begin{array}{lll}
\boldsymbol{g} & \omega_{\text {ie }} & \boldsymbol{g} \times \omega_{i e}
\end{array}\right] \\
& \boldsymbol{B}_{2}=\left[\begin{array}{lll}
\boldsymbol{g} & \boldsymbol{g} \times \omega_{\text {ie }} & \left(\boldsymbol{g} \times \omega_{i e}\right) \times \boldsymbol{g}
\end{array}\right]
\end{aligned}
$$

It is quite obvious from (4) and (5) that $\boldsymbol{B}_{1}$ and $\boldsymbol{B}_{2}$ are suitable for analytical alignment except at the Earth poles. The transformation matrix $C_{b}^{n}$ can be directly obtained from (3) as:

$$
C_{b}^{n}=\left[\begin{array}{lll}
V_{1} & V_{2} & V_{3}
\end{array}\right]_{n}\left[\begin{array}{lll}
V_{1} & V_{2} & V_{3}
\end{array}\right]_{b}
$$

Alternatively, since $C_{b}^{n}$ is orthogonal, it must satisfy:

$$
\left(C_{b}^{n}\right)^{-1}=\left(C_{b}^{n}\right)^{T}
$$

Therefore $C_{b}^{n}$ can also be written as:

$$
C_{b}^{n}=\left[\begin{array}{l}
\left(V_{1}^{n}\right)^{T} \\
\left(V_{2}^{n}\right)^{T} \\
\left(V_{3}^{n}\right)^{T}
\end{array}\right]^{-1}\left[\begin{array}{l}
\left(V_{1}^{b}\right)^{T} \\
\left(V_{2}^{b}\right)^{T} \\
\left(V_{3}^{b}\right)^{T}
\end{array}\right]
$$

By substituting the three vectors of $\boldsymbol{B}_{1}$ into (8), $C_{b}^{n}$ becomes [2]:

$$
C_{b}^{n}=\left[\begin{array}{c}
\left(g^{n}\right)^{T} \\
\left(\omega_{i e}^{n}\right)^{T} \\
\left(g^{n} \times \omega_{i e}^{n}\right)^{T}
\end{array}\right]^{-1}\left[\begin{array}{c}
\left(g^{b}\right)^{T} \\
\left(\omega_{i e}^{b}\right)^{T} \\
\left(g^{b} \times \omega_{i e}^{b}\right)^{T}
\end{array}\right]
$$

Let $\boldsymbol{a}^{\boldsymbol{b}}$ and $\omega^{\boldsymbol{b}}$ represent the measurements of accelerometers and gyros, respectively, and define their components in the body frame as: 


$$
\begin{aligned}
\boldsymbol{a}^{\boldsymbol{b}} & =\left[\begin{array}{lll}
a_{x} & a_{y} & a_{z}
\end{array}\right]^{T} \\
\boldsymbol{\omega}^{\boldsymbol{b}} & =\left[\begin{array}{lll}
\omega_{x} & \omega_{y} & \omega_{z}
\end{array}\right]^{T}
\end{aligned}
$$

In practical implementations, $\boldsymbol{g}^{\boldsymbol{b}}$ and $\boldsymbol{\omega}_{\boldsymbol{i}}^{\boldsymbol{b}}$ are replaced by $-\boldsymbol{a}^{\boldsymbol{b}}$ and $\boldsymbol{\omega}^{\boldsymbol{b}}$ respectively. Then using $(1,2,10,11)$ the element of $C_{b}^{n}$ in (9) can be explicitly expressed as [5,6].

$$
C_{b}^{n}=\left[\begin{array}{ccc}
\frac{g a_{x}-a_{x} \Omega \sin \varphi}{g \Omega \cos \varphi} & \frac{g a_{y}-a_{y} \Omega \sin \varphi}{g \Omega \cos \varphi} & \frac{g a_{z}-a_{z} \Omega \sin \varphi}{g \Omega \cos \varphi} \\
\frac{a_{z} a_{y}-a_{y} a_{z}}{g \Omega \cos \varphi} & \frac{a_{x} a_{z}-a_{z} a_{x}}{g \Omega \cos \varphi} & \frac{a_{y} a_{x}-a_{x} a_{y}}{g \Omega \cos \varphi} \\
\frac{-a_{x}}{g} & \frac{-a_{y}}{g} & \frac{-a_{z}}{g}
\end{array}\right]
$$

Similarly, if the three vectors in $\boldsymbol{B}_{2}$ are employed, then $C_{b}^{n}$ in (9) can be written as:

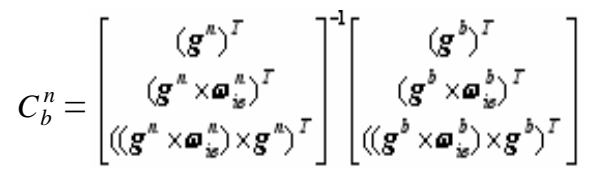

In this case, the element of second and third rows of $C_{b}^{n}$ is the same as (12), whereas the elements of first row are changed to:

$$
\begin{aligned}
& C_{11}=a^{2} \omega_{x}-a_{x}\left(\mathbf{a}^{b} \cdot \omega^{b}\right) /\left(g^{2} \Omega \cos \varphi\right) \\
& C_{12}=a^{2} \omega_{y}-a_{y}\left(\mathbf{a}^{b} \cdot \omega^{b}\right) /\left(g^{2} \Omega \cos \varphi\right) \\
& C_{13}=a^{2} \omega_{z}-a_{z}\left(\mathbf{a}^{b} \cdot \omega^{b}\right) /\left(g^{2} \Omega \cos \varphi\right)
\end{aligned}
$$

Where $a$ is the magnitude of $\boldsymbol{a}^{\boldsymbol{b}}$. We can easily see that the elements of the first row in (12) and (14) are identical by using the equalities, $a=g$ and $\boldsymbol{a}^{\boldsymbol{b}} \cdot \boldsymbol{\omega}^{\boldsymbol{b}}=g \Omega \sin \varphi$ in the ideal situation. However, their error characteristics are quite different.

\section{ERROR ANALYSIS}

In the error analysis, we now consider a vector $\boldsymbol{V}$, which is known in the navigation frame and can be obtained by processing the sensor outputs. Ideally, their relationship can be written as [9]:

$$
V^{n}=C_{b}^{n} V^{b}
$$

where $C_{b}^{n}$ represents the true transformation matrix. However, it is inevitable that the inertial sensing signals will be contaminated with uncertainties in a practical strap down system. Therefore, only the computational transformation matrix $\hat{C}_{b}^{n}$ and the noisy vector $\hat{\boldsymbol{V}}^{\boldsymbol{b}}$ are available. At the end of alignment process, the relationship (15) has to be rewritten as:

$$
\boldsymbol{V}^{\boldsymbol{n}}=\hat{C}_{b}^{n} \boldsymbol{V}^{\boldsymbol{b}}
$$


When the initial alignment process has been completed, $\hat{C}_{b}^{n}$ and $C_{b}^{n}$ can be related by the following formula [2]:

$$
\hat{C}_{b}^{n}=(I+S-\phi) C_{b}^{n}
$$

Where $I$ is the identity matrix while both $S$ and $\phi$ represent the alignment errors. The matrix $S$ is symmetric with the form of:

$$
S=\left[\begin{array}{lll}
S_{N} & \varepsilon_{D} & \varepsilon_{E} \\
\varepsilon_{D} & S_{E} & \varepsilon_{N} \\
\varepsilon_{E} & \varepsilon_{N} & S_{D}
\end{array}\right]
$$

Where the diagonal elements $S_{i}, \mathrm{i}=\mathrm{N}, \mathrm{E}, \mathrm{D}$, represent the scale factor errors and the offdiagonal elements $\varepsilon_{i}, \mathrm{i}=\mathrm{N}, \mathrm{E}, \mathrm{D}$, represent the skew misalignment angles. The matrix $\phi$ is anti symmetrical with the form of:

$$
\phi=\left[\begin{array}{ccc}
0 & -\phi_{D} & \phi_{E} \\
\phi_{D} & 0 & -\phi_{N} \\
-\phi_{E} & \phi_{N} & 0
\end{array}\right]
$$

Where the elements $\phi_{i}, \mathrm{i}=\mathrm{N}, \mathrm{E}, \mathrm{D}$, denote the drift misalignment angles. Moreover, $\boldsymbol{V}^{\boldsymbol{b}}$ is perturbed as:

$$
\hat{\boldsymbol{V}}^{\boldsymbol{b}}=\boldsymbol{V}^{\boldsymbol{b}}+\delta \boldsymbol{V}^{\boldsymbol{b}}
$$

Substituting (17) and (20) into (16), it can be found that, to the first order approximation,

$$
(S-\phi) \boldsymbol{V}^{\boldsymbol{n}}=-C_{b}^{n} \delta \boldsymbol{V}^{\boldsymbol{b}}
$$

This is the basic error equation for analysis of analytic alignment methods. In order to analyze the alignment error associated with the computational of method-1 in (9) we can individually substitute the three vectors of $\boldsymbol{B}_{1}$ in (4) for $\boldsymbol{V}$ in (21).

Firstly, assume that $\boldsymbol{V}=\boldsymbol{g}$ and use the fact that $\delta \boldsymbol{g}^{\boldsymbol{b}}=-\delta \boldsymbol{a}^{\boldsymbol{b}}$ with simple manipulation equation (21) can be rewritten as:

$$
(S-\phi) \boldsymbol{g}^{\boldsymbol{n}}=-C_{b}^{n} \delta \boldsymbol{g}^{\boldsymbol{b}}=C_{b}^{n} \delta \boldsymbol{a}^{\boldsymbol{b}}
$$

Secondly, assume that $\boldsymbol{V}=\boldsymbol{\omega}_{i e}$ and use the fact that $\delta \boldsymbol{\omega}_{i e}=\delta \boldsymbol{\omega}^{b}$ with simple manipulation equation (21) leads to:

$$
(S-\phi) \boldsymbol{\omega}_{i e}=-C_{b}^{n} \delta \boldsymbol{\omega}_{i e}^{b}=-C_{b}^{n} \delta \boldsymbol{\omega}^{b}
$$

Finally, assume that $\boldsymbol{V}=\boldsymbol{g} \times \boldsymbol{\omega}_{i e}$ and apply the fact that $\delta \mathbf{V}^{\boldsymbol{b}}=-\delta \boldsymbol{a}^{\boldsymbol{b}} \times \boldsymbol{\omega}_{\boldsymbol{i e}}^{\boldsymbol{b}}+\boldsymbol{g}^{\boldsymbol{b}} \times \delta \boldsymbol{\omega}^{\boldsymbol{b}}$ thus, equation (21) leads to:

$$
\begin{aligned}
& (\mathrm{S}-\phi)\left(\boldsymbol{g} \times \omega_{i c}^{n}\right)=-\mathrm{C}_{b}^{n}\left(-\delta \boldsymbol{a}^{b} \times \omega_{i c}^{b}+\boldsymbol{g}^{b} \times \delta \omega^{b}\right) \\
& =\left(\mathrm{C}_{b}^{n} \delta \boldsymbol{a}^{b}\right) \times \omega_{i c}^{n}-\boldsymbol{g}^{n} \times\left(\mathrm{C}_{b}^{n} \delta \omega^{b}\right)
\end{aligned}
$$


Denoting the effective sensing errors in the navigation frame as:

$$
\begin{aligned}
& \delta \boldsymbol{a}^{n}=C_{\boldsymbol{b}}^{\boldsymbol{n}} \delta \boldsymbol{a}^{\boldsymbol{b}}=\left[\begin{array}{lll}
\delta \mathrm{a}_{N} & \delta \mathrm{a}_{\boldsymbol{E}} & \delta \mathrm{a}_{\boldsymbol{D}}
\end{array}\right]^{T} \\
& \delta \boldsymbol{\omega}^{n}=C_{b}^{n} \delta \boldsymbol{\omega}^{b}=\left[\begin{array}{lll}
\delta \omega_{N} & \delta \omega_{E} & \delta \omega_{D}
\end{array}\right]^{T}
\end{aligned}
$$

It means that, in general, the alignment errors are composed of the entire strap down sensing errors. Now substituting $(1,2,18,19,25,26)$ into (22) yields the set of these equations:

$$
\begin{aligned}
& \delta a_{N}=g\left(\varepsilon_{E}-\phi_{E}\right) \\
& \left(\varepsilon_{E}-\phi_{E}\right)=\frac{\delta a_{N}}{g} \\
& \delta a_{E}=g\left(\varepsilon_{N}+\phi_{N}\right) \\
& \left(\varepsilon_{N}+\phi_{N}\right)=\frac{\delta a_{E}}{g} \\
& \delta a_{D}=g S_{D} \\
& S_{D}=\frac{\delta a_{D}}{g}
\end{aligned}
$$

Substituting $(1,2,18,19,25,26)$ into (23) yields the set of these equations:

$$
\begin{aligned}
& \delta \omega_{N}=\Omega \sin \varphi\left(\varepsilon_{E}-\phi_{E}\right)-S_{N} \Omega \cos \varphi \\
& S_{N}=\frac{\delta a_{N}}{g} \tan \varphi-\frac{\delta \omega_{N}}{\Omega \cos \varphi} \\
& \left(\varepsilon_{D}-\phi_{D}\right)=\left(\varepsilon_{N}+\phi_{N}\right) \tan \varphi-\frac{\delta \omega_{E}}{\Omega \cos \varphi} \\
& \delta \omega_{D}=\Omega \sin \varphi S_{D}-\Omega \cos \varphi\left(\varepsilon_{E}+\phi_{E}\right) \\
& \left(\varepsilon_{E}+\phi_{E}\right)=S_{D} \tan \varphi-\frac{\delta \omega_{D}}{\Omega \cos \varphi}
\end{aligned}
$$

Substituting $(1,2,18,19,25,26)$ into (24) yields the set of these equations:

$$
\begin{aligned}
& \left(\varepsilon_{D}+\phi_{D}\right)=\frac{\delta \omega_{E}}{\Omega \cos \varphi}-\frac{\delta a_{E}}{g} \tan \varphi \\
& g \Omega \cos \varphi S_{E}=-g \hat{n} \omega_{N}+\Omega \sin \varphi \delta a_{N}+\Omega \cos \varphi \omega_{D}^{n} \\
& S_{f}=\frac{\delta a_{D}}{g}+\frac{\delta a_{N}}{g} \tan \varphi-\frac{\delta \omega_{1}}{\Omega \cos \varphi} \\
& \delta a_{E}=-g\left(\varepsilon_{N}-\phi_{N}\right) \\
& \left(\varepsilon_{N}-\phi_{N}\right)=-\frac{\delta a_{E}}{g}
\end{aligned}
$$

Adding equation (35) and (28), the value of $\varepsilon_{N}$ tends to be zero and substituting in equation (28), the value of $\phi_{N}$ can be found. Adding equation (27) and equation (32), we can determine the value of $\varepsilon_{E}$ then substituting in equation (27). Thus, the value of $\phi_{E}$ can be 
calculated. Adding Eq. (31) with (33) we found that the value of $\varepsilon_{D}$ tends to be zero and then, substituting in Eq. (33) the value of $\phi_{D}$ can be found.

Finally, the analytical alignment errors can be uniquely determined. The drift misalignment angles can be obtained as:

$$
\begin{aligned}
& \phi_{N}=\frac{\delta a_{E}}{g} \\
& \phi_{E}=\frac{1}{2}\left(-\frac{\delta a_{N}}{g}+\frac{\delta a_{D} \tan \varphi}{g}-\frac{\delta \omega_{D}}{\Omega \cos \varphi}\right) \\
& \phi_{D}=\frac{\delta \omega_{E}}{\Omega \cos \varphi}-\frac{\delta a_{E} \tan \varphi}{g}
\end{aligned}
$$

These drifts had been presented in the literature [2]. It is observed from (37) that not only the north accelerometer error but also the vertical accelerometer and azimuth gyro uncertainties will include the east level error. This situation is quite different than that occurred in a gimbaled system. It should be noted that an orthonormalization process does not have effect on these drift errors. Although an orthonormalization process can eliminate the skew and scale factor errors, they are also presented here for completeness. The skew misalignment angles can be obtained as:

$$
\begin{aligned}
& \varepsilon_{D}=\varepsilon_{N}=0 \\
& \varepsilon_{E}=\frac{1}{2}\left(\frac{\delta a_{N}}{g}+\frac{\delta a_{D}}{g} \tan \varphi-\frac{\delta \omega_{D}}{\Omega \cos \varphi}\right)
\end{aligned}
$$

which shows that, the east skew error dose not equals zero.

The scale errors are given by:

$$
\begin{aligned}
& S_{N}=\frac{\delta a_{N}}{g} \tan \varphi-\frac{\delta \omega_{N}}{\Omega \cos \varphi} \\
& S_{E}=\frac{\delta a_{D}}{g}+\frac{\delta a_{N}}{g} \tan \varphi-\frac{\delta \omega_{N}}{\Omega \cos \varphi} \\
& S_{D}=\frac{\delta a_{D}}{g}
\end{aligned}
$$

Similarly, the alignment errors associated with the computational method in (13) can be obtained by substituting the three vectors of $\boldsymbol{B}_{2}$ in (5) for $\boldsymbol{V}$. After simple manipulations, the drift misalignment angles can be obtained as:

$$
\begin{aligned}
& \phi_{N}=\frac{\delta a_{E}}{g} \\
& \phi_{E}=-\frac{\delta a_{N}}{g} \\
& \phi_{D}=\frac{\delta \omega_{E}}{\Omega \cos \varphi}-\frac{\delta a_{E} \tan \varphi}{g}
\end{aligned}
$$

It is obvious that the drift errors, (44-46) are identical to those obtained with physical gyro compassing. All of the skew misalignment angles are identically zero, i.e. 
$\varepsilon_{N}=\varepsilon_{E}=\varepsilon_{D}=0$,

and the scale factor errors are given by

$$
\begin{aligned}
& S_{N}=2 \frac{\delta a_{D}}{g}+\frac{\delta a_{N}}{g} \tan \varphi-\frac{\delta \omega_{N}}{\Omega \cos \varphi} \\
& S_{E}=\frac{\delta a_{D}}{g}+\frac{\delta a_{N}}{g} \tan \varphi-\frac{\delta \omega_{N}}{\Omega \cos \varphi} \\
& S_{D}=\frac{\delta a_{D}}{g}
\end{aligned}
$$

As compared with (38), (46) is not corrupted by vertical accelerometer and azimuth gyro uncertainties. In practice, the azimuth gyro uncertainty will dominate the east level error of (38). Hence, the computational of method-2 in (13) is better than that given in (9).

\section{SIMULATION RESULTS}

A simulation program is carried out for verification purposes. Extensive simulation results are at various parameters are conducted. The following case study is chosen to be presented here. The following parameters are utilized while the drift misalignment angles are resulted: Latitude angle $\varphi=30^{\circ}$; pitch angle $\theta=30^{\circ}$; yaw angle $\psi=-45^{\circ}$; roll angle $\gamma=10^{\circ}$.

The sensor error is chosen constant as: gyro drift $\varepsilon=0.1^{0} / \mathrm{h}$, accelerometer bias $\nabla=100 \mu \mathrm{g}$; measurement noise $\sigma_{\varepsilon}=0.01^{\circ} / \mathrm{h}$, and $\sigma_{\nabla}=10 \mu \mathrm{g}$, total time $1 \mathrm{~min}$ and sampling time $0.1 \mathrm{sec}$.

The two matrices $\phi_{1}$ and $\phi_{2}$ represents the drift misalignment angles by using method1 and method-2 are recorded as follows:

$$
\begin{aligned}
\phi_{1} & =\left[\begin{array}{ccc}
-41.7653 & 11.7968 & -12.8336 \\
-11.7965 & -41.5984 & -0.1509 \\
0.2008 & 0.1285 & 0.1792
\end{array}\right] \\
\phi_{2} & =\left[\begin{array}{ccc}
-41.4254 & 11.7965 & 0.5494 \\
-11.7965 & -41.5984 & -0.1509 \\
-0.5489 & 0.1527 & 0.1731
\end{array}\right]
\end{aligned}
$$

The two matrices $S_{1}$ and $S_{2}$ represents the scale factor errors and skew misalignment angles by method- 1 and method- 2 as follows:

$$
\begin{aligned}
& S_{1}=\left[\begin{array}{ccc}
-41.7917 & 0.0000 & -6.3166 \\
0.0000 & -41.6187 & 0.0000 \\
-6.3166 & 0.0000 & 0.1730
\end{array}\right] \\
& S_{2}=\left[\begin{array}{ccc}
-41.4457 & 0.0000 & 0.0000 \\
0.0000 & -41.6187 & 0.0000 \\
0.0000 & 0.0000 & 0.1730
\end{array}\right]
\end{aligned}
$$


Table $(1,2)$ shows the drift misalignment angles by using both proposed methods and the estimated attitude angles via the coarse alignment:

Table 1. Drift misalignment angles

\begin{tabular}{|l|c|c|c|}
\hline & $\phi_{\mathrm{N}}[\mathrm{min}]$ & $\phi_{\mathrm{E}}[\mathrm{min}]$ & $\phi_{\mathrm{D}}[\mathrm{min}]$ \\
\hline Method 1 & -0.1541 & -6.8651 & -11.7442 \\
\hline Method 2 & -0.1541 & $-0,5485$ & -11.7442 \\
\hline
\end{tabular}

Table 2. Estimated attitude angles

\begin{tabular}{|l|c|c|c|}
\hline & Yaw [deg] & Pitch [deg] & Roll [deg] \\
\hline Method 1 & -44.8007 & 30.0753 & 9.9095 \\
\hline Method 2 & -44.8487 & 29.9917 & 10.0054 \\
\hline Actual & -45 & 30 & 10 \\
\hline
\end{tabular}

\section{CONCLUSIONS}

A few general requirements for analytic self-alignment are considered. Two useful computation methods of coarse alignment for strap down inertial navigation systems are presented. Their associated drift, skew, and scale alignment errors are evaluated analytically. Although the computational formulas for analytic ground alignment are identical in the ideal case, the error characteristics are dependent upon the employed basis. The alignment computational method 2 is superior to method 1 practically because its east level error is not corrupted by gyro uncertainty. Moreover, the drift misalignment angles of method 2 are equivalent to those obtained with physical gyro-compassing. These results are helpful in design of ground coarse alignment process.

\section{REFERENCES}

[1] Siouris, G.M. (1987) Navigation, Inertial. Encyclopedia of physical Science and Technology, 8 (1987), 668-717.

[2] Mortensen, R.E. (1974) Strapdown guidance error analysis. IEEE Transaction on Aerospace and Electronic System, AES-10, 4 (July 1974),451-457.

[3] JANG Gyu Lee, Chan Gook Park, Ileung Park, "Multi-position Alignment of Strap-down Inertial Navigation System. IEEE Transaction on Aerospace and Electronic System, Vol. 29, No. 4 Oct. 1999.

[4] Yeon Fuh Jiang, Chung Shan. "Error Analysis of Analytic Coarse Alignment Methods", IEEE Transaction on Aerospace and Electronic System, Vol. 34, No.1 Jan. 2001.

[5] Britting, K.R. (1971), "Inertial Navigation System Analyses". New York: Wiley Interscience, 1971. Ch.9.

[6] Stieler, B., and Winter, H. (1982), "Gyroscopic instruments and their application to flight testing". AGARD Dograph 160, 15 (1982), ch.7.

[7] Nebot Eduardo, Durrant-Whyte Hugh. Department of Mechanical and Mechatronic Engineering University of Sydney, 2006,NSW,Australia,0-8186-8023/97IEEE 1997. 
[9] Dohyoung Chung, Jang Gyu Lee, Chan Gook Park, Heung Won Park (Strap down INS Error Model For Multiposition Alignment) IEEE Transactions on aerospace and electronic systems Vol. 32, No. 4 October 2001 